\title{
PROPERTIES OF HIGHLY POROUS CORDIERITE CERAMIC OBTAINED BY DIRECT FOAMING AND GELCASTING METHOD
}

\author{
YE LI*, WEI CAO*, LUNLUN GONG*, RUIFANG ZHANG*, "XUDONG CHENG*, ** \\ *State Key Laboratory of Fire Science, University of Science and Technology of China, \\ Hefei, Anhui 230027, PR China \\ **Collaborative Innovation Center for Urban Public Safety, \\ Anhui Province, PR China \\ ${ }^{\#}$ E-mail: chengxd@ustc.edu.cn
}

Submitted September 7, 2015; accepted February 23, 2016

\begin{abstract}
Keywords: Porous ceramic, Cordierite, Porosity, Thermal conductivity, Sintering degree
The highly porous cordierite ceramics were fabricated combining the merits of direct foaming and gelcasting method. The content of gum arabic had a great influence on the foaming capacity and the stability of the wet slurry foam. And the foaming capacity mainly affected the porosity while the stability mainly affected the pore size. The highest porosity of $90 \%$ was obtained at $0.4 \mathrm{wt} . \%$ gum arabic content and the smallest pore size and the most homogeneous pore distribution were arrived at $1.2 \mathrm{wt} . \%$ gum arabic content. As the sintering temperature increased, the porosity and pore size decreased but the uniformity of the pore distribution increased. Moreover, the thermal conductivity was mainly affected not only by the porosity, but also by the sintering degree of the cordierite substrate.
\end{abstract}

\section{INTRODUCTION}

Cordierite foam ceramics have many unique properties such as excellent thermal shock resistance, high melting point, high corrosion resistance, low thermal conductivity, low density, and low dielectric constant [1-4]. The performance of porous ceramics mainly depends on the porosity, pore size, morphology and distribution, all of which are determined by the processing steps involved in the fabrication of the ceramic foams. Numerous processing methods have been developed to produce ceramic foams [5-8]. In previous work, we fabricated the porous cordierite [9] and anorthite [10] ceramics using the direct foaming method and the slip casting method. The viscosity of the slurry, porosity, density and pore structure were studied in detail. And the relationship between porosity and thermal conductivity was discussed based on Gong's model [11]. However, the slip casting method has its own disadvantages, such as cracks, low strength and porosity gradient in the sintered samples. All of these impedes the further study of the above factors affecting the thermal conductivity. Therefore, it is necessary to find another solidification method to avoid the above defects in the samples. Gelcasting process combined with foaming method has been used to produce porous ceramics [12-14]. When the slurry foams are prepared, gelcasting process can maintain the porous structure effectively before the sintering process. Moreover, the mechanical strength of sintered foams is higher than those obtained by other routes [15] and the samples have a more homogeneous pore microstructure.
In this study, the rheological behavior, porosity, microstructure, thermal conductivity at room temperature, and pore size distribution were investigated. Moreover, the experimental thermal conductivity data were compared with theoretical values derived from Gong's model. The factors affecting the thermal conductivity, including porosity, pore size, pore distribution and sintering degree were also discussed.

\section{EXPERIMENTAL}

Commercial cordierite powder (Hebei, China) was used to fabricate the porous ceramic. Gum arabic $(\mathrm{Cp}$, Shanghai, China) was added as the dispersant. 0.5 wt. \% content of agar ( $\mathrm{Cp}$, Shanghai, China) based on the solid phase was used to solidify the slurry. The dodecyl sodium sulfate (K12, Cp, Shanghai, China) was added into the slurry as the foaming agent.

40 wt. \% concentrated slurry was chosen to prepare the green bodies. For this purpose, the mixture and different amounts of dispersant were added into deionized water and ball-milled for $24 \mathrm{~h}$ at a rotation speed of $180 \mathrm{rpm}$. Then the slurry was poured into a beaker and heated to $60^{\circ} \mathrm{C}$ by water bath. Meanwhile, 0.5 wt. $\%$ content of agar was heated to $90^{\circ} \mathrm{C}$ to dissolve in water. Afterwards, both of them with $\mathrm{K} 12$ were mixed and stirred using a direct driven motor at a speed of $1500 \mathrm{rpm}$ to produce wet slurry foams. After stirring for $3 \mathrm{~min}$, the wet slurry foams were cast into the molds fabricated by silicone. Subsequently, the molds were dried at room temperature for $48 \mathrm{~h}$ before samples removal. Then the 
wet green bodies were dried at room temperature for $24 \mathrm{~h}$ and in a drying oven at $80^{\circ} \mathrm{C}$ for $24 \mathrm{~h}$. After drying, the specimens were sintered between 1200 and $1300^{\circ} \mathrm{C}$ at an interval of $50^{\circ} \mathrm{C}$ for $2 \mathrm{~h}$. A detailed flowchart of the process is shown in Figure 1.

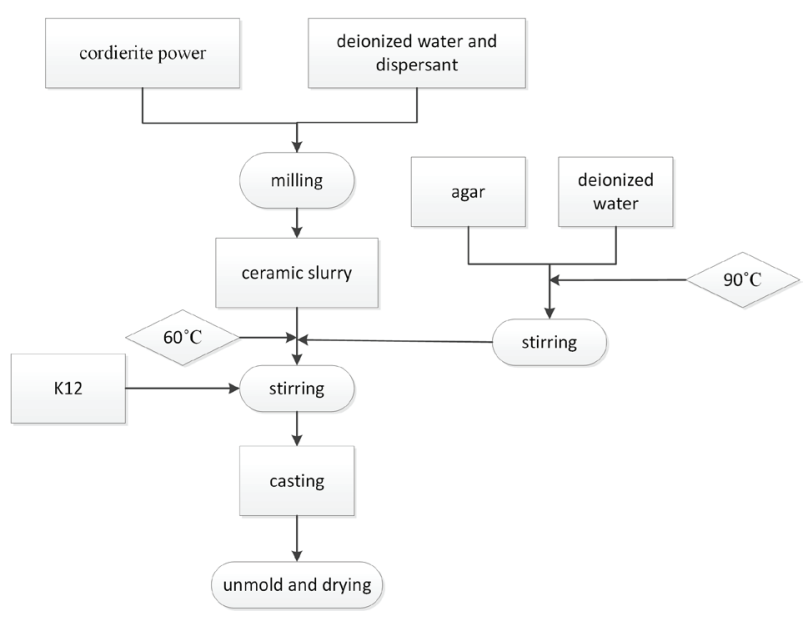

Figure 1. Detailed flowchart of the process used to prepare porous green bodies.

The viscosity of the slurry was measured using NDJ-5S rotational viscometer. The foaming capacity was determined by the ratio of the height after and before foaming, which was simply measured by vernier caliper. The microstructure was observed on FEI Quanta FEG 450 scanning electron microscopy (SEM). The pore size distribution was obtained by analyzing SEM micrographs using an image analyzer (Nano measurer, China) with a total of at least 200 pores being counted on each image. The open porosity was determined by the Archimedes method using distilled water as liquid medium. And the thermal conductivity of the samples was measured by DRE-2C thermal conductivity tester.

\section{RESULTS AND DISCUSSION}

Viscosity and foaming capacity

The rheological behavior has a great influence on the foaming capacity and the stability of wet slurry foam. Therefore, the variation of the slurry viscosity is an important part of this research. Figure 2 shows the effect of the gum arabic contents on the viscosity of the slurry. For all the samples with different gum arabic contents, the viscosity of the slurry was not high and the slurries showed pseudoplastic flow behavior, which could favor the generation of the foam due to the lower viscosity under shearing [16]. As the content of gum arabic increased, the viscosity decreased first and then increased. The 0.4 wt. \% content of gum arabic correlated with the optimum concentration for complete coverage of the dispersant onto the ceramic particles creating a repulsive potential, resulting in a low-viscosity slip [17]. The further increase in viscosity occurred as a result of an interaction between the excess free polymer chains in the suspension [17]. Generally, the effect of the viscosity on the foaming capacity directly reflects in the foaming volume/height, which also determines the porosity [18]. The ratio of the height after and before foaming was represented in Figure 3. The curves of the slurry foaming capacity were in good agreement with the viscosity curves, and at 0.4 wt. \% content of the gum arabic, the foaming capacity of the slurry was the highest. After foaming, the viscosity of the slurry foam had a substantial increase (as shown in Figure 4), which can be attributed to the presence of bubbles and surfactant molecules at the gas-liquid interfaces $[13,16]$. Moreover, the viscosity of the slurry after foaming had the similar variation to the results shown in Figure 2. But the minimum value of the viscosity appeared when the content of the gum arabic was 0.8 wt. $\%$ rather than 0.4 wt. $\%$. This is the result of the interaction, including the dispersant which decreased the viscosity and the foaming process increasing the viscosity.

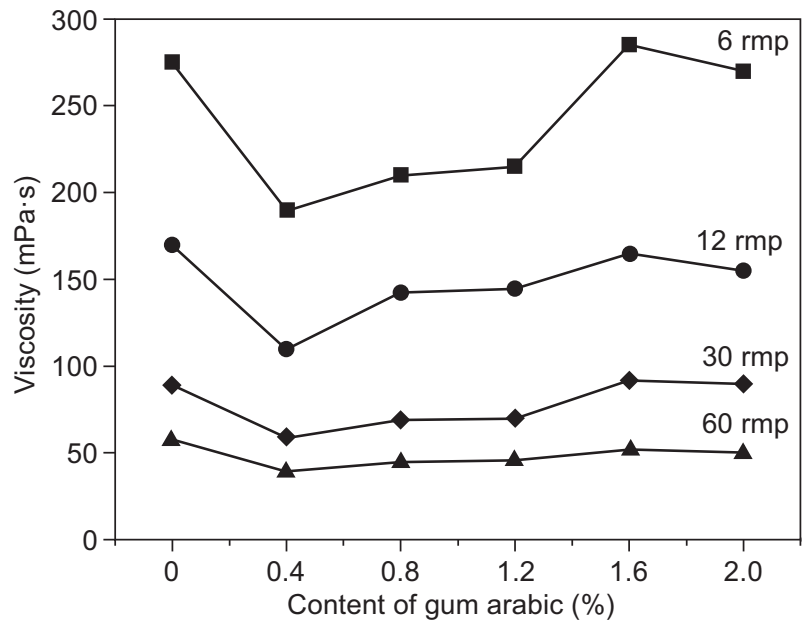

Figure 2. Viscosity variation with different contents of gum arabic before foaming.

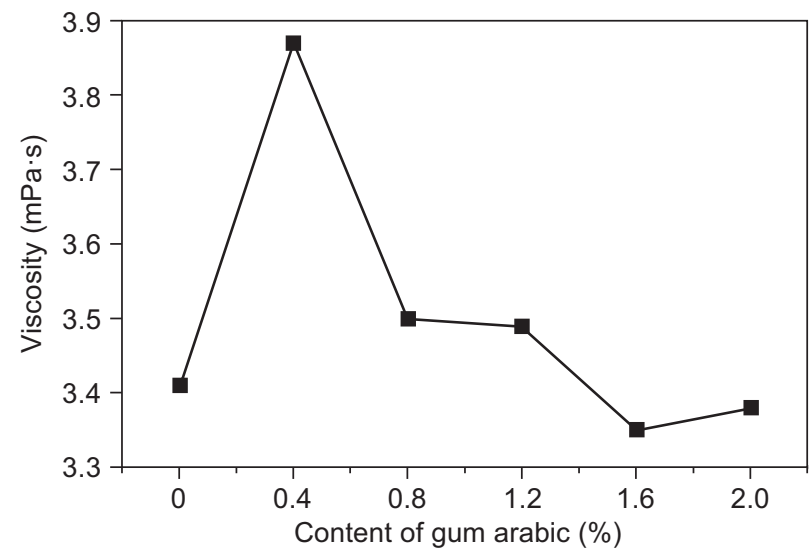

Figure 3. Foaming capacity of the slurry with different contents of gum arabic. 


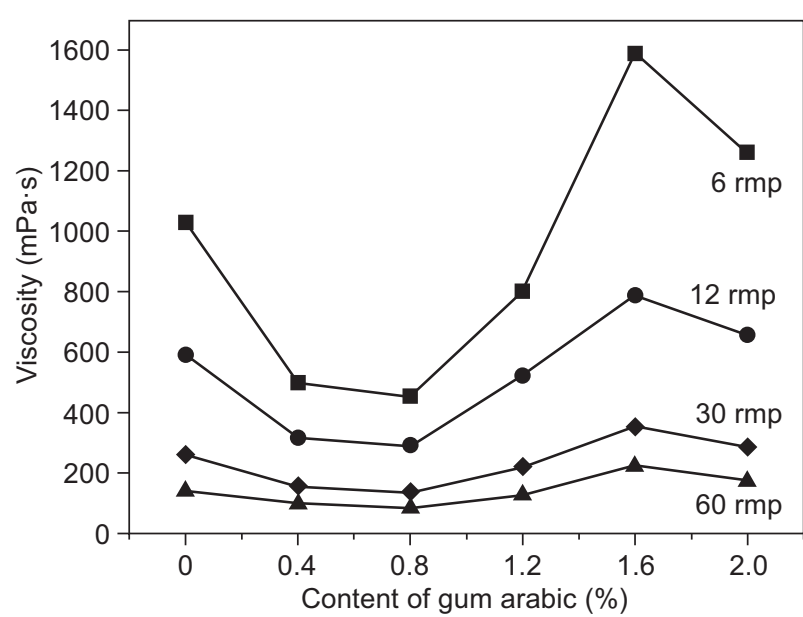

Figure 4. Viscosity variation with different contents of gum arabic after foaming.

\section{Porosity}

Figure 5 shows the total porosity and open porosity of samples varied as a function of gum arabic content. The total porosity of sintered sample is calculated from the theoretical fully density of cordierite ceramic. For all the sintering temperatures, the total porosity and open porosity varied in a small range with different gum arabic contents. However, it is still obvious that for all the samples, both the total porosity and open porosity increased first and then decreased with the increasing content of the gum arabic, which is similar to the trend of the foaming capacity (as shown in Figure 3). This indicates that the foaming capacity rather than the stability of the foamed slurry dominates the total porosity and open porosity of the samples. Also, the maximum values of the total porosity and open porosity appeared at 0.4 wt. $\%$ content of the gum arabic. Moreover, a gradual decrease in total porosity and open porosity with

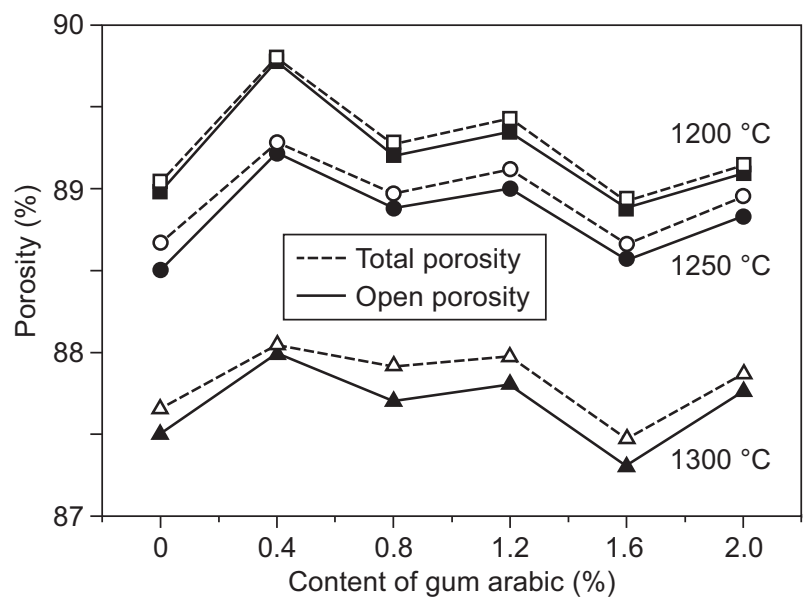

Figure 5. Total porosity and open porosity of samples with different gum arabic contents sintered at $1200-1300^{\circ} \mathrm{C}$. temperature was observed for all the samples with the same gum arabic doping levels. It can be concluded that the effect of the temperature on the total porosity and open porosity is prior to the effect of the viscosity in this work. The closed porosity, which is the difference value between total porosity and open porosity, increased as the temperature increased from $1200^{\circ} \mathrm{C}$ to $1300^{\circ} \mathrm{C}$, indicating that part of open pores transformed into the closed pores during sintering process.

\section{Microstructure}

Figure 6 presents the SEM microphotographs of the samples with $0-2.0$ wt. \% gum arabic contents sintered at $1250^{\circ} \mathrm{C}$. The samples with different dispersant contents exhibited similar tri-modal pore structure with macropores and windows. The macropores and windows were believed to form during the foaming process [19]. The windows connecting the two different cells caused a high open porosity. As shown in Figure 7, the maximum value of pore size appeared when the gum arabic content was 0.4 wt. $\%$. And the variation of the pore size curve was in contrast to the results of the viscosity shown in Figure 2 and 4, which can be ascribed to the stability of the slurry during the foaming process and gelation process. This suggests that the pore size was determined mainly by the stability of the slurry rather than the foaming capacity. The stability is mainly manifested in two aspects. On the one hand, it is difficult to break up the bubbles to be smaller by the stirring paddle in the process of foaming when the viscosity is high. On the other hand, the higher viscosity restrains the growth and coalescence of the bubbles to be larger in the gelling process. Figure 8 shows the pore size distributions of samples with different gum arabic contents sintered at $1250^{\circ} \mathrm{C}$. All of the curves were unimodal, indicating that the pore size distributions of the samples were uniform. When the content of the gum arabic was $1.2 \mathrm{wt} . \%$, the pore size distribution had the best uniformity.

Figure 9 shows the SEM microphotographs of the samples sintered at different temperatures without Gum arabic added. As the temperature incerased from 1200 to $1300^{\circ} \mathrm{C}$, the average pore size decreased from 230 to $190 \mu \mathrm{m}$ (shown in Figure 10). This can be ascribed to the shrinkage of the pores and the densification caused by the high temperature. Moreover, based on the slopes of the pore size curves between different temperatures, the reducing rate of the pore size increased with the temperature increase. Figure 11 presents the pore distributions of samples sintered at different temperatures. The uniformity of pore distribution also decreased with the increasing temperature due to the shrinkage and densification of the samples. This is an unavoidable process that the transformation of the pore diameter from large to small will lead up to the nonuniformity of the pore distribution [10]. 


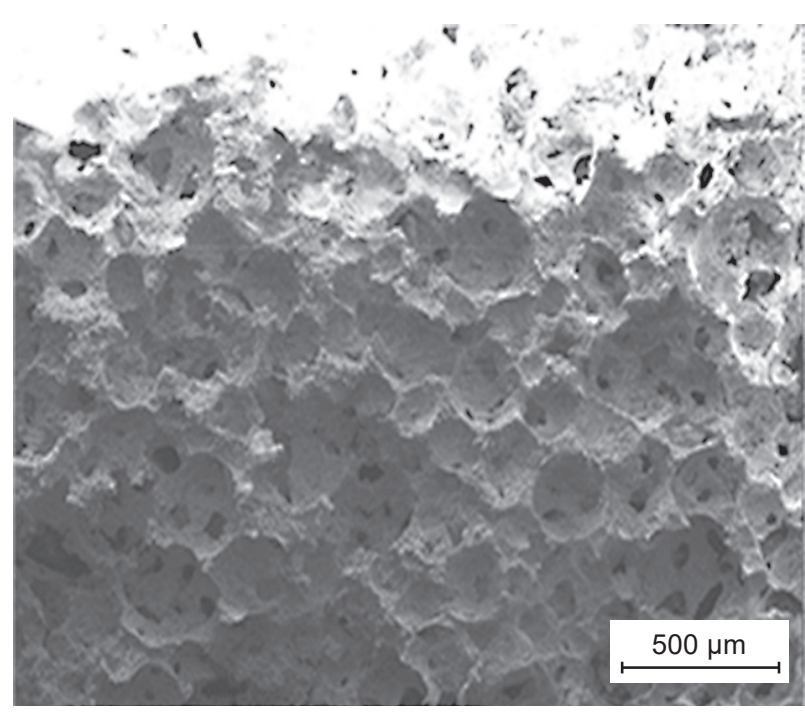

a) 0 wt. $\%$

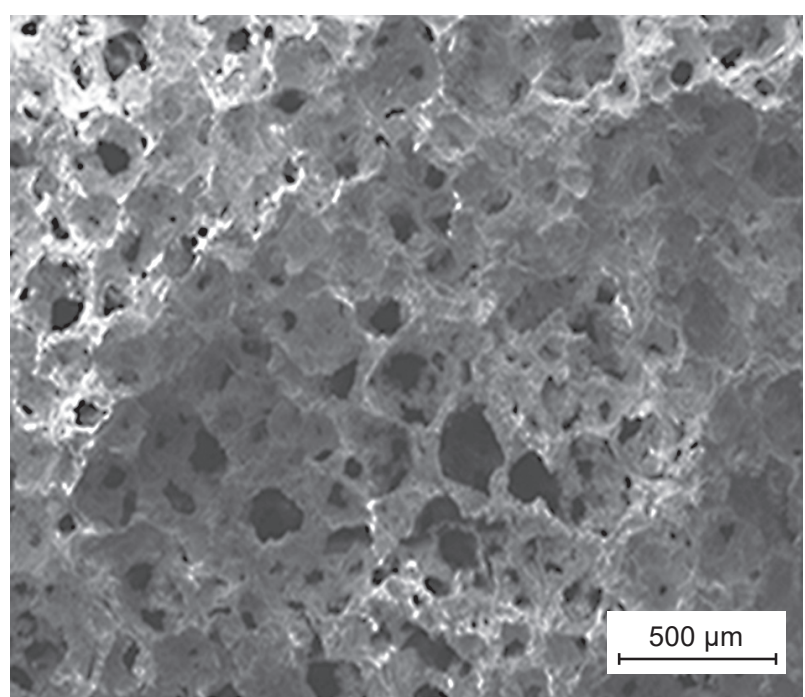

c) 0.8 wt. \%

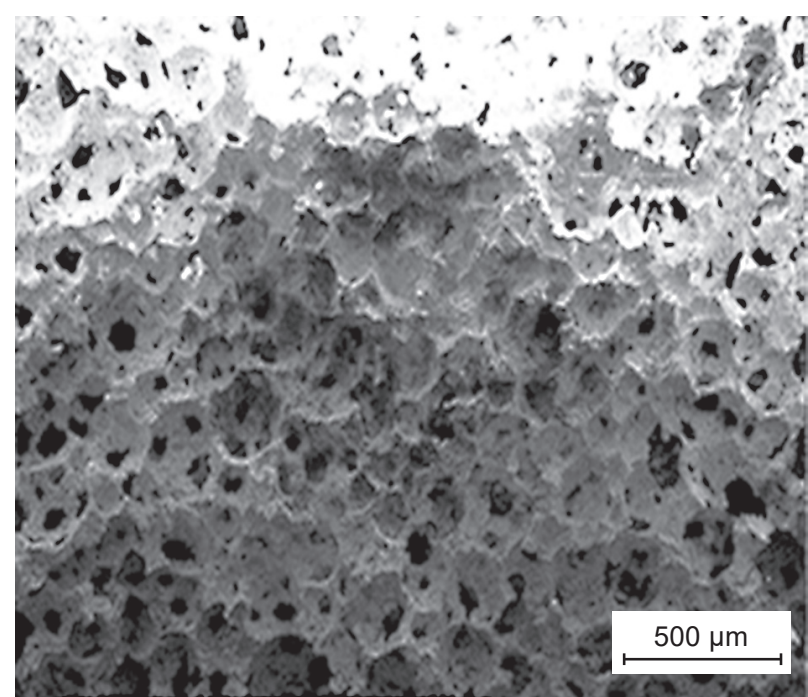

e) 1.6 wt. $\%$

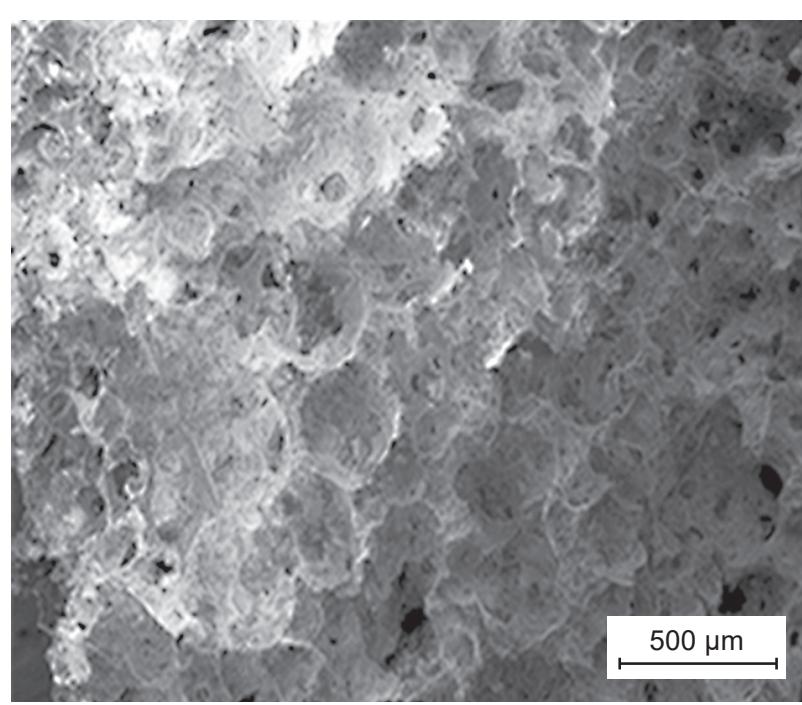

b) 0.4 wt. $\%$

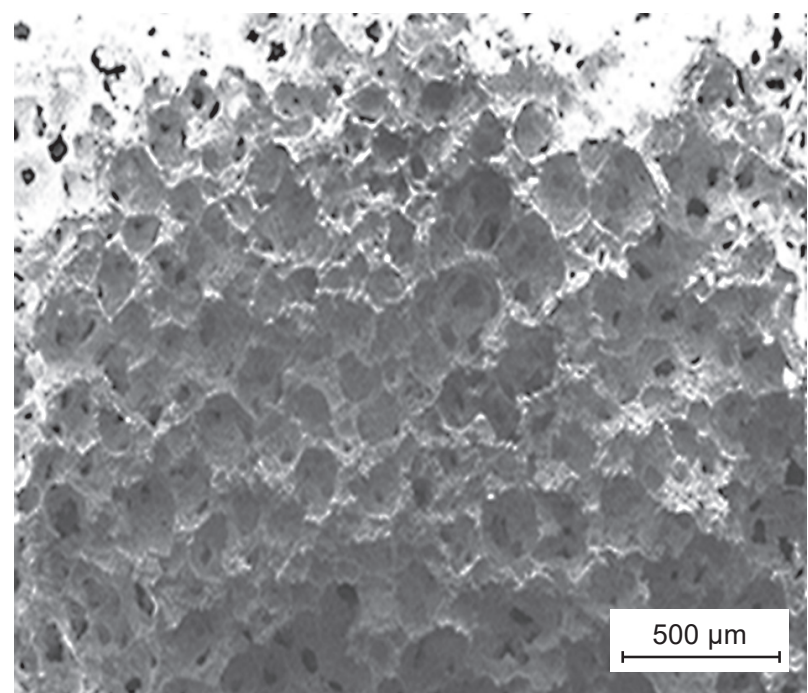

d) 1.2 wt. $\%$

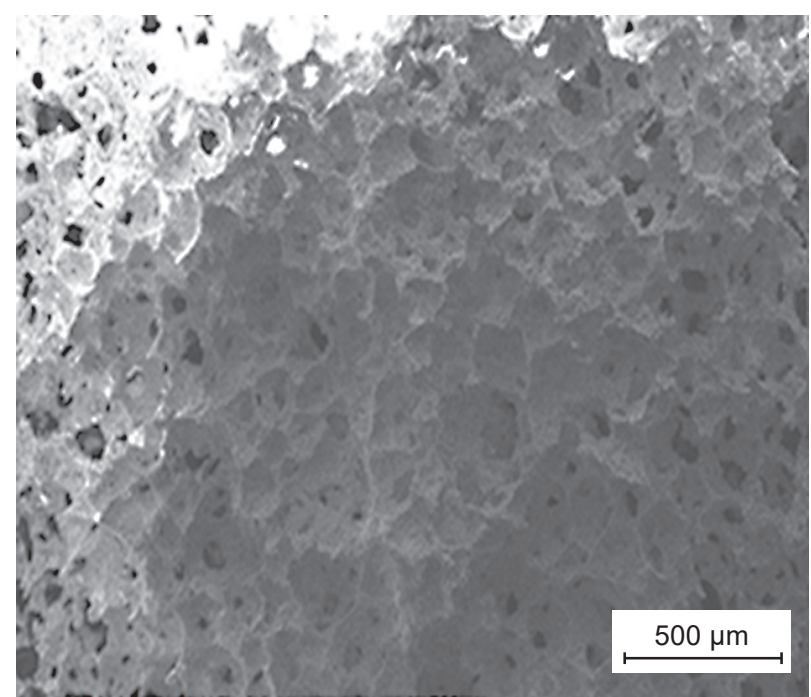

f) 2.0 wt. $\%$

Figure 6. SEM microphotographs of the samples with $0-2.0$ wt. $\%$ gum arabic contents sintered at $1250^{\circ} \mathrm{C}$ : a) 0 wt. $\%$; b) 0.4 wt. $\%$; c) 0.8 wt. $\%$; d) 1.2 wt. $\%$; e) 1.6 wt. $\%$; f) 2.0 wt. $\%$. 


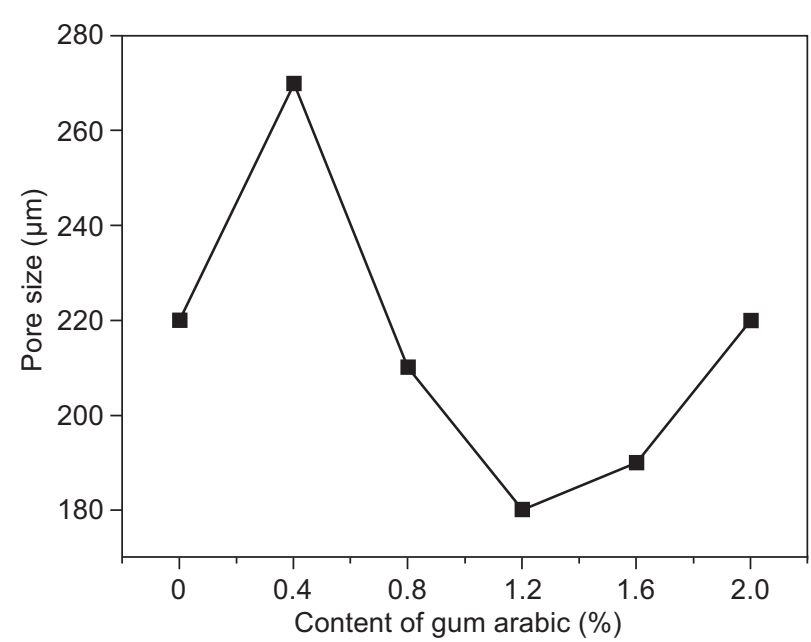

Figure 7. Average pore size variation with different gum arabic contents sintered at $1250^{\circ} \mathrm{C}$.

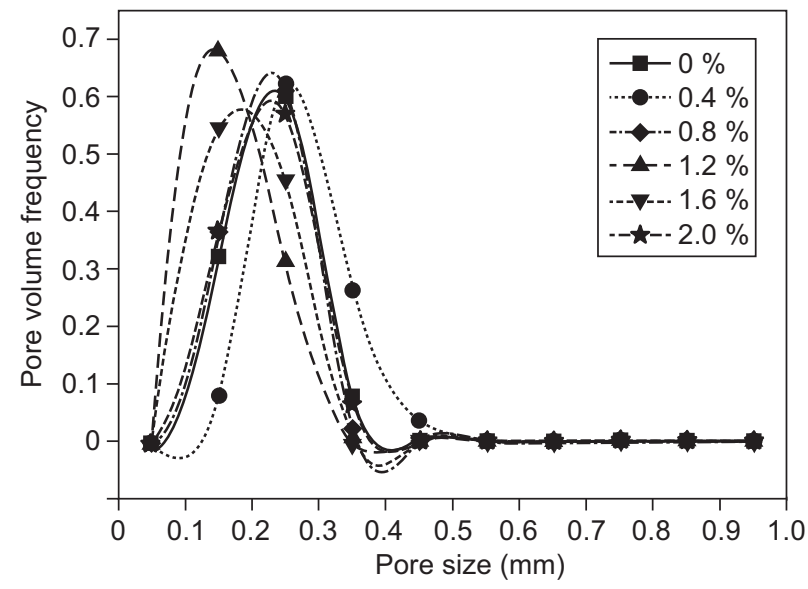

Figure 8. Pore size distributions of samples with different gum arabic contents sintered at $1250^{\circ} \mathrm{C}$.

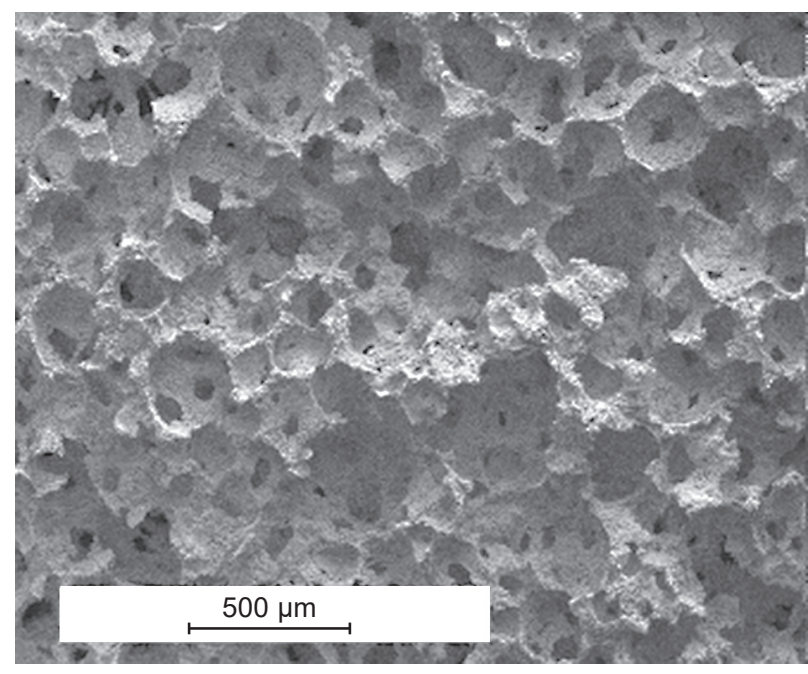

a) $1200^{\circ} \mathrm{C}$

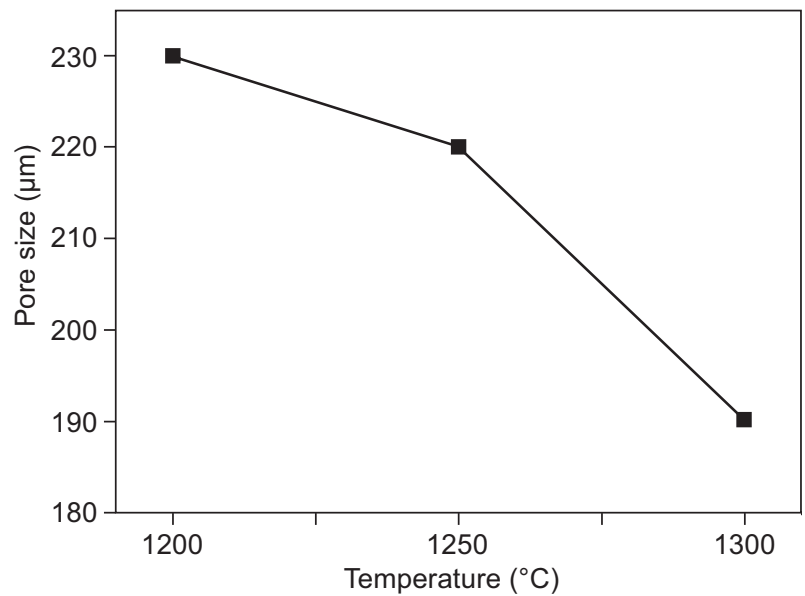

Figure 10. Average pore size variation with different sintering temperatures.

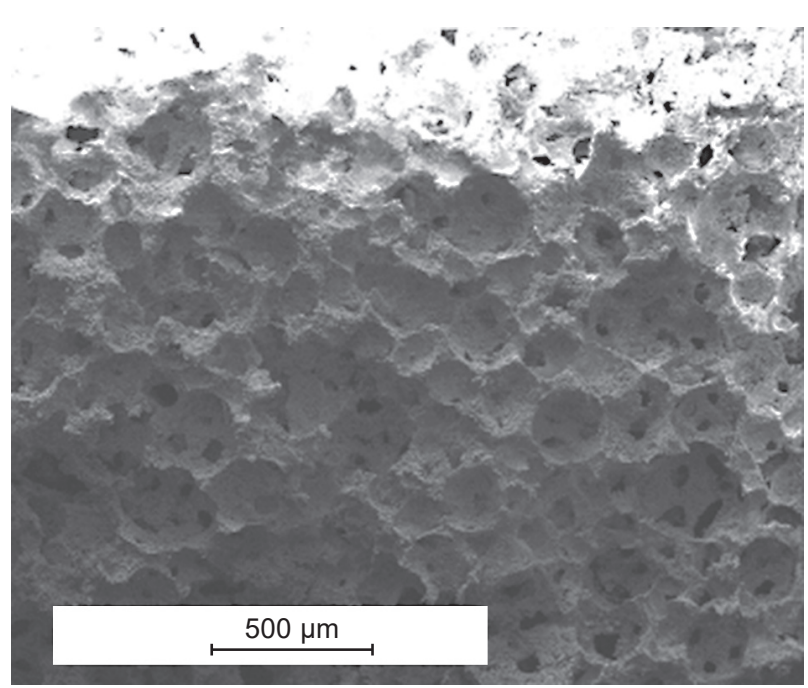

b) $1250^{\circ} \mathrm{C}$

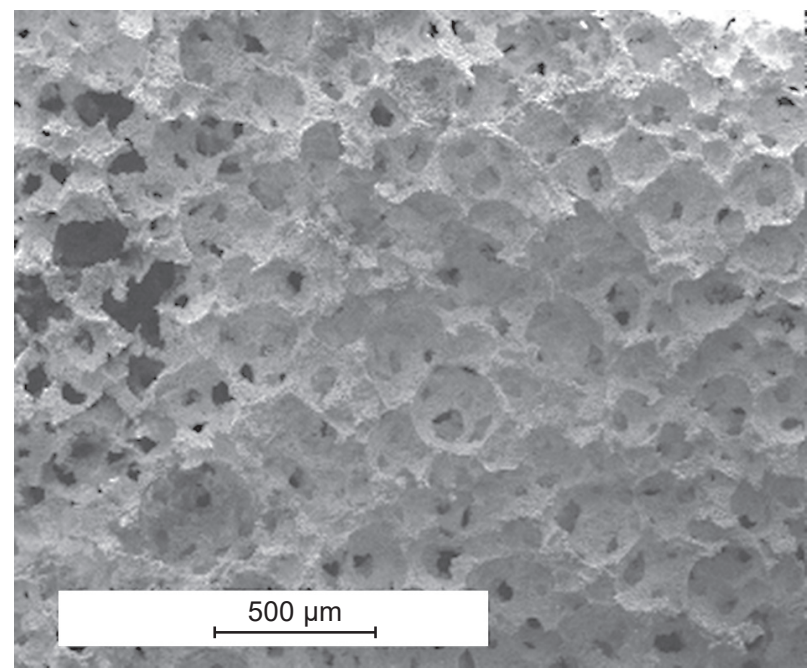

c) $1300^{\circ} \mathrm{C}$

Figure 9. SEM microphotographs of the samples sintered at different temperatures: a) $1200^{\circ} \mathrm{C}$; b) $1250^{\circ} \mathrm{C}$; c) $1300^{\circ} \mathrm{C}$. 


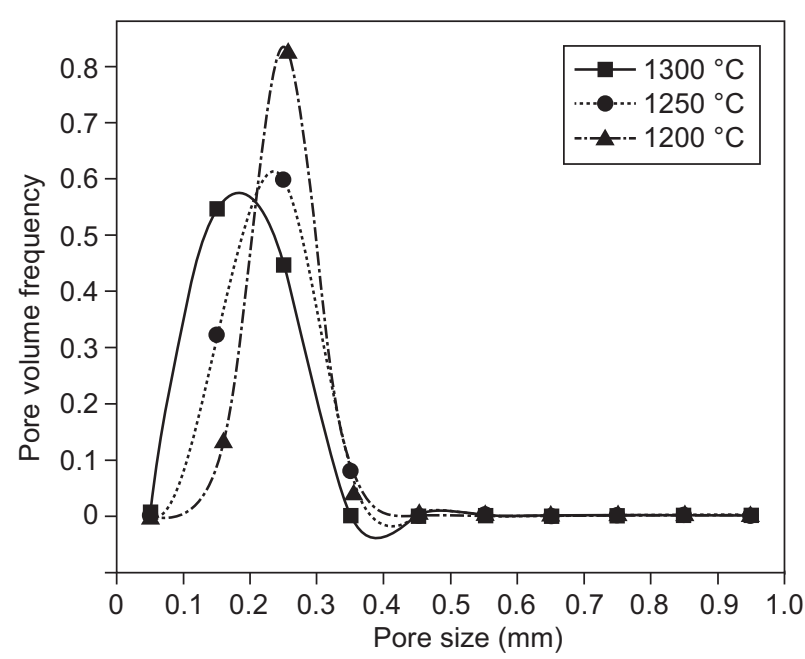

Figure 11. Pore size distributions of samples sintered at different temperatures.

\section{Thermal conductivity}

The thermal conductivity measurement was carried out by TPS technique at room temperature [20]. The main advantages of the hot disk technique include wide thermal conductivity range $\left(0.005-500 \mathrm{~W} \cdot \mathrm{m}^{-1} \cdot \mathrm{K}^{-1}\right)$, wide range of materials types, easy sample preparation, nondestructive measurement and high accuracy [21]. The measured results show that the thermal conductivity of the porous cordierite ceramics increased from 0.06 to $0.12 \mathrm{~W} \cdot \mathrm{m}^{-1} \cdot \mathrm{K}^{-1}$ with porosity decreasing from 90 to $87 \%$. Thus it can be found that though the variation of porosity was slight for the samples, the corresponding thermal conductivity had a large scope. Figure 12 presents the thermal conductivity as a function of porosity for the experimental data. For all the samples sintered at different temperatures, the thermal conductivity decreased as the porosity increased. And it is obvious that the distribution of the thermal conductivity data presented three levels based on the temperature.

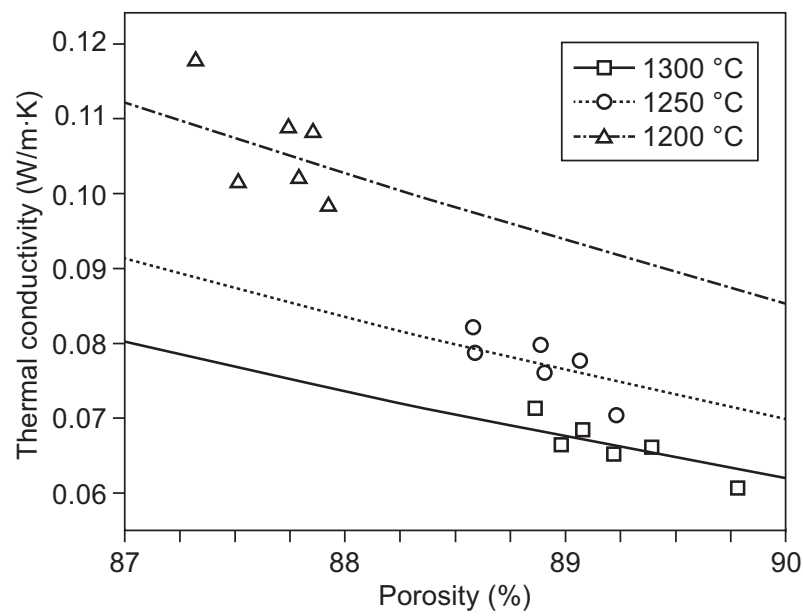

Figure 12. Thermal conductivity as a function of porosity for the experimental data and Gong's model curves.
As a general rule, a porous material can be considered as a two-phase system, viz. a dense solid skeleton and air [11]. For the porous cordierite ceramics prepared in this work, they consist of a dense cordierite skeleton and air. Based on this assumption, there are many researches on the relation between thermal conductivity and porosity, and some prediction models have been put forward, including the Series and Parallel models [22], Maxwell-Eucken models [23, 24], EMT equation $[25,26]$, a universal model [27] and so on. As shown in Figure 12, the thermal conductivity curves of the porous cordierite ceramic were plotted based on the Gong's model, which had been used in the porous mullite and anorthite ceramics successfully $[10,11]$. In fact, Gong's model is a semi-empirical model, which can be defined as follows.

$$
\sum_{\mathrm{i}=1}^{\mathrm{w}} v_{i} \frac{k_{i}-k_{e}}{k_{i}+2 \chi k_{e}}=0
$$

where $k$ and $v$ are thermal conductivity and volume fraction, respectively. A proportionality coefficient $\chi$ is added, which is related to the microstructure of porous materials. The formula of $\chi$ can be obtained by fitting parts of experimental data, and the usual expression is given.

$$
\chi=\exp (a+b \varepsilon)+1
$$

where $\varepsilon$ is porosity. The effect of the microstructure on the thermal conductivity may reflect on the impact of $a$ and $b$.

It can be found that the three curves represented the variation of thermal conductivity related to the porosity at three different sintering temperatures. However, the porosity is not the only factor that affects the thermal conductivity. Pore size, pore structure and grain size also had obvious effects on heat conduction [28]. Small pore size and uniform pore distribution both contributed to lower thermal conductivity [10, 29]. Based on the results in section 3.3 and Figure 12, it can be observed that the sintering degree was the other factor greatly affecting the thermal conductivity rather than pore size, pore distribution or grain size. As the sintering temperature increased, larger amount of particles connected with each other, which led to the stronger sintering necks and increasingly strong network in the sintered bodies, increasing the heat transfer channel. In fact, for the two-phase system, both of the two phases (gas phase and solid phase) varied with the sintering process while the change of the solid skeleton was not reflected in the above models.

\section{CONCLUSIONS}

In the present work, highly porous cordierite ceramics with porosity from 87 to $90 \%$ were fabricated combining the merits of direct foaming and gelcasting method. The gum arabic content had a great influence on the foaming capacity and the stability of the wet slurry 
foam. Moreover, it can be found that the foaming capacity mainly affected the porosity while the stability mainly affected the pore size. At 0.4 wt. \% gum arabic content, the highest porosity of $90 \%$ was obtained and at $1.2 \mathrm{wt}$. \% gum arabic content the samples had the smallest pore size and the most homogeneous pore distribution. As the sintering temperature increased, the porosity and pore size decreased but the uniformity of the pore distribution increased due to the shrinkage and densification of the samples. Moreover, the distribution of the thermal conductivity data presented three different levels based on the sintering temperature. And the porosity continued to dominate the thermal conductivity but the sintering degree rather than pore size or pore distribution also had a great influence on the thermal conductivity in this paper. However, for the two-phase system, both of the two phases varied with the sintering process while the change of the solid skeleton was not reflected in all of the existing models, which should be further studied.

\section{Acknowledgements}

This work was supported by the National Basic Research Program of China (973 Program) (2012CB 719701), and National Key Technology R\&D Program of China (2013BAJ01B05) and Fundamental Research Funds for the Central Universities (WK2320000032).

\section{REFERENCES}

1. Liu D.M. (1998): Preparation and characterisation of porous hydroxyapatite bioceramic via a slip-casting route. Ceramics International, 24(6), 441-446. doi:10.1016/ S0272-8842(97)00033-3

2. Hirschfeld D.A., Li T.K., Liu D.M. (1995). Processing of porous oxide ceramics. Key Engineering Materials, 115, 65-80. doi:10.4028/www.scientific.net/KEM.115.65

3. Wang H., Li X., Hong L.Y., Kim D.P. (2006): Preparation of phenolic resin derived 3-D ordered macroporous carbon. Journal of Porous Materials, 13(2), 115-121. doi:10.1007/ s10934-006-7006-9

4. Montanaro L., Jorand Y., Fantozzi G., Negro A. (1998): Ceramic foams by powder processing. Journal of the European Ceramic Society, 18(9), 1339-1350. doi:10.1016/ S0955-2219(98)00063-6

5. Nor M.A.A.M., Hong L.C., Ahmad Z.A., Akil H.M. (2008): Preparation and characterization of ceramic foam produced via polymeric foam replication method. Journal of Materials Processing Technology, 207(1), 235-239. doi:10.1016/j.jmatprotec.2007.12.099

6. Andersson L., Bergström L. (2008): Gas-filled microspheres as an expandable sacrificial template for direct casting of complex-shaped macroporous ceramics. Journal of the European Ceramic Society, 28(15), 2815-2821. doi:10.1016/ j.jeurceramsoc.2008.04.020

7. Barg S., Soltmann C., Andrade M., Koch D., Grathwohl G. (2008): Cellular ceramics by direct foaming of emulsified ceramic powder suspensions. Journal of the American
Ceramic Society, 91(9), 2823-2829. doi: 10.1111/j.15512916.2008.02553.x

8. Colombo P., Hellmann J.R., Shelleman D.L. (2001): Mechanical properties of silicon oxycarbide ceramic foams. Journal of the American Ceramic Society, 84(10), 22452251. doi:10.1111/j.1151-2916.2001.tb00996.x

9. Li Y., Cao W., Feng J., Gong L., Cheng X. (2015): Fabrication of cordierite foam ceramics using direct foaming and slip casting method with plaster moulds. Advances in Applied Ceramics, 114(8), 465-470. doi: 10.1179/1743676115Y.0000000022

10. Li Y., Cheng X., Gong L., Feng J., Cao W., Zhang R., Zhang H. (2015): Fabrication and characterization of anorthite foam ceramics having low thermal conductivity. Journal of the European Ceramic Society, 35(1), 267-275. doi:10.1016/j.jeurceramsoc.2014.08.045

11. Gong L., Wang Y., Cheng X., Zhang R., Zhang H. (2013): Thermal conductivity of highly porous mullite materials. International Journal of Heat and Mass Transfer, 67, 253259. doi:10.1016/j.ijheatmasstransfer.2013.08.008

12. Yuan B., Wu H., Sun X., Wang G., Li H. (2012): Fabrication of porous alumina green bodies from suspension emulsions by gelcasting. Materials Letters, 81, 151-154. doi:10.1016/j. matlet.2012.04.112

13. Mao X., Shimai S., Wang S. (2008): Gelcasting of alumina foams consolidated by epoxy resin. Journal of the European Ceramic Society, 28(1), 217-222. doi:10.1016/j. jeurceramsoc.2007.06.006

14. Tomita T., Kawasaki S., Okada K. (2005): Effect of viscosity on preparation of foamed silica ceramics by a rapid gelation foaming method. Journal of Porous Materials, 12(2), 123 129. doi:10.1007/s10934-005-6769-8

15. Sepulveda P., Ortega F.S., Innocentini M.D., Pandolfelli V.C. (2000): Properties of highly porous hydroxyapatite obtained by the gelcasting of foams. Journal of the American Ceramic Society, 83(12), 3021-3024. doi: 10.1111/j.11512916.2000.tb01677.x

16. Sepulveda P., Binner J.G.P. (1999): Processing of cellular ceramics by foaming and in situ polymerisation of organic monomers. Journal of the European Ceramic Society, 19(12), 2059-2066. doi:10.1016/S0955-2219(99)00024-2

17. Cyster L.A., Grant D.M., Howdle S.M., Rose F.R.A.J., Irvine D.J., Freeman D., Scotchford C.A., Shakesheff K. M. (2005): The influence of dispersant concentration on the pore morphology of hydroxyapatite ceramics for bone tissue engineering. Biomaterials, 26(7), 697-702. doi:10.1016/j.biomaterials.2004.03.017

18. Wang M., Du H., Guo A., Hao R., Hou Z. (2012): Microstructure control in ceramic foams via mixed cationic/anionic surfactant. Materials Letters, 88, 97-100. doi:10.1016/j.matlet.2012.08.028

19. Han Y., Choi J., Kim H.S., Kim H., Park J. (2013): Control of pore and window size of ceramic foams with tri-modal pore structure: Influence of agar concentration. Materials Letters, 110, 256-259. doi:10.1016/j.matlet.2013.05.100

20. Solorzano E., Rodriguez-Perez M.A., Reglero J.A., De Saja J.A. (2007): Density gradients in aluminium foams: characterisation by computed tomography and measurements of the effective thermal conductivity. Journal of Materials Science, 42(8), 2557-2564. doi:10.1007/s10853006-1233-y

21. He Y. (2005): Rapid thermal conductivity measurement with a hot disk sensor. Thermochimica Acta, 436(1-2), 122-129. doi:10.1016/j.tca.2005.06.026 
22. Singh R., Kasana H.S. (2004): Computational aspects of effective thermal conductivity of highly porous metal foams. Applied thermal engineering, 24(13), 1841-1849. doi:10.1016/j.applthermaleng.2003.12.011

23. Hashin Z.,Shtrikman S., (1962): A Variational Approach to the Theory of the Effective Magnetic Permeability of Multiphase Materials. Journal of Applied Physics, 33(10), 3125-3131. doi:10.1063/1.1728579

24. Clerk M.J. (1954). A treatise on electricity and magnetism. $3^{\text {rd }}$ ed.: Dover Publications Inc., New York.

25. Landauer R. (1952). The electrical resistance of binary metallic mixtures. Journal of Applied Physics, 23(7), 779784. doi:10.1063/1.1702301
26. Eucken A. (1940): Allgemeine gesetzmäßigkeiten für das wärmeleitvermögen verschiedener stoffarten und aggregatzustände. Forschung auf dem Gebiet des Ingenieurwesens A, 11(1), 6-20.

27. Brailsford A.D., Major K.G. (1964): The thermal conductivity of aggregates of several phases, including porous materials. British Journal of Applied Physics, 15, 313-322.

28. Han Y., Li C., Bian C., Li S., Wang C.-A. (2013: Porous anorthite ceramics with ultra-low thermal conductivity. Journal of the European Ceramic Society, 33(13-14), 25732578. doi:10.1016/j.jeurceramsoc.2013.04.006

29. Nait-Ali B., Haberko K., Vesteghem H., Absi J., Smith D. S. (2006): Thermal conductivity of highly porous zirconia. Journal of the European Ceramic Society, 26(16), 35673574. doi:10.1016/j.jeurceramsoc.2005.11.011 